\title{
Corrigendum: Pioglitazone for NAFLD Patients With Prediabetes or Type 2 Diabetes Mellitus: A Meta-Analysis
}

\author{
Jingxuan Lian and Jianfang $F u^{*}$ \\ Department of Endocrinology, Xijing Hospital of Air Force Medical University, Xi'an, China
}

\section{OPEN ACCESS}

Approved by: Frontiers Editorial Office, Frontiers Media SA, Switzerland

${ }^{*}$ Correspondence: Jianfang Fu

Jianf@fmmu.edu.cn

Specialty section: This article was submitted to Clinical Diabetes, a section of the journal Frontiers in Endocrinology

Received: 21 December 2021 Accepted: 24 January 2022

Published: 16 February 2022

Citation: Lian J and Fu J (2022) Corrigendum: Pioglitazone for NAFLD Patients With

Prediabetes or Type 2 Diabetes Mellitus: A Meta-Analysis. Front. Endocrinol. 13:840299. doi: 10.3389/fendo.2022.840299
Keywords: NAFLD, type 2 diabetes mellitus, pioglitazone, meta-analysis, prediabetes

\section{A Corrigendum on}

Pioglitazone for NAFLD Patients With Prediabetes or Type 2 Diabetes Mellitus: A MetaAnalysis

by Lian, J. and Fu, J. (2021). Front. Endocrinol. 12:615409. doi: 10.3389/fendo.2021.615409

In the article as originally published the labeling of the forest plots shown in Figures $\mathbf{3 A - F}$ were not correct. The use and positioning of the text "favours [pioglitazone]" and "favours [placebo]" seemed to imply that the placebo group had a higher risk of morbidity. A correct version of the Figure 3, with updated labeling is shown here.

The authors apologize for this error and state that this does not change the scientific conclusions of the article in any way. The original article has been updated.

\footnotetext{
Publisher's Note: All claims expressed in this article are solely those of the authors and do not necessarily represent those of their affiliated organizations, or those of the publisher, the editors and the reviewers. Any product that may be evaluated in this article, or claim that may be made by its manufacturer, is not guaranteed or endorsed by the publisher.

Copyright $(2022$ Lian and Fu. This is an open-access article distributed under the terms of the Creative Commons Attribution License (CC BY). The use, distribution or reproduction in other forums is permitted, provided the original author(s) and the copyright owner(s) are credited and that the original publication in this journal is cited, in accordance with accepted academic practice. No use, distribution or reproduction is permitted which does not comply with these terms.
} 
A

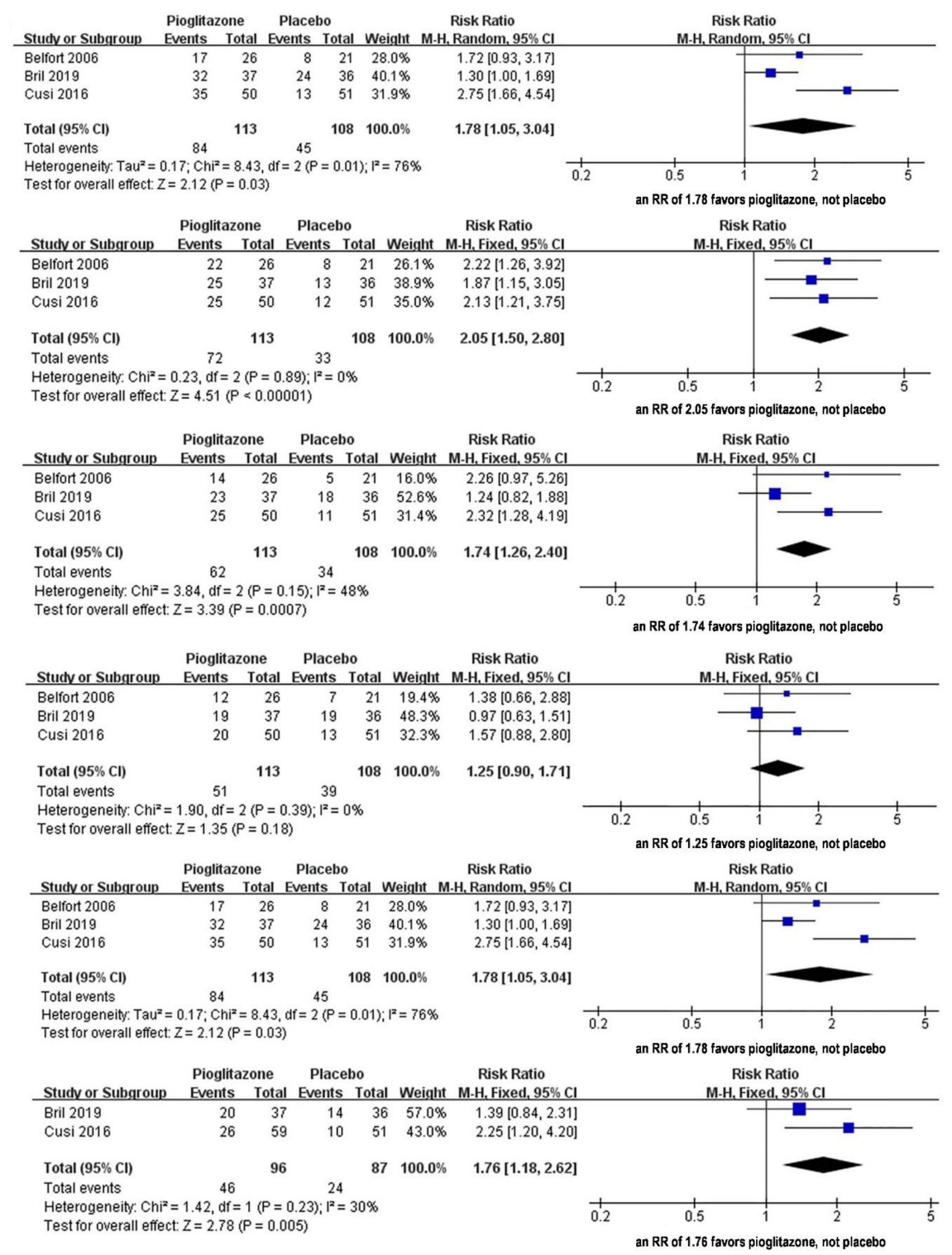

FIGURE 3 | The effect of pioglitazone in hepatic histologic scores (steatosis grade) with improvement of at least 1 grade (A). The effect of pioglitazone in hepatic histologic scores (inflammation grade) with improvement of at least 1 grade (B). The effect of pioglitazone in hepatic histologic scores (ballooning grade) with improvement of at least 1 grade (C). The effect of pioglitazone in hepatic histologic scores (fibrosis stage) with improvement of at least 1 grade (D). The effect of pioglitazone in resolution of NASH without worsening of fibrosis (E). The effect of pioglitazone in reduction of at least 2 points in hepatic histologic scores (from two different parameters) (F). 